\title{
Towards all-numerical implementation of correlation
}

\author{
A. Alfalou ${ }^{1, *}$, C. Brosseau ${ }^{2}$, B. - E. Benkelfat ${ }^{3}$, S. Qasmi ${ }^{1}$, and I. Léonard ${ }^{1}$ \\ ${ }^{1}$ ISEN Brest, Groupe Vision, L@ bISEN, 20 rue Cuirassé Bretagne, CS 42807, \\ 29228 Brest Cedex 2, France. \\ ayman.al-falou@isen.fr \\ ${ }^{2}$ Université Européenne de Bretagne, Université de Brest, Lab-STICC (UMR CNRS 6185), \\ CS 93837, 6 avenue Le Gorgeu, 29238 Brest Cedex 3, France. \\ ${ }^{3}$ Institut Télécom -Télécom Sud Paris, SAMOVAR (UMR CNRS-INT 5157), \\ Département Electronique et Physique, 9 rue Charles Fourier, 91011 Evry Cedex, France.
}

\begin{abstract}
Interestingly, the past 20 years have provided us many examples of optical correlation methods for pattern recognition, e.g. VanderLugt correlator (VLC). In recent years, hybrid techniques, i.e. numerical implementation of correlation, have been also considered an alternative to all-optical methods because they show a good compromise between performance and simplicity. Moreover, these correlation methods can be implemented using an all-numerical and reprogrammable target such as the graphics processor unit (GPU), or the field-programmable gate array (FPGA). However, this numerical procedure requires realizing two Fourier Transforms (FT), a spectral multiplication, and a correlation plane analysis. The purpose of this study is to compare the performances of a numerical correlator based on the fast Fourier transform (FFT) with that relying on a simulation of the Fraunhofer diffraction. Different tests using the Pointing Head Pose Image Database (PHPID) and considering faces with vertical and horizontal rotations were performed with the code MATLAB. Tests were conducted with a five reference optimized composite filter. The receiving operating characteristics (ROC) curves show that the optical FT simulating the Fraunhofer diffraction leads to better performances than the FFT. The implications of our results for correlation are discussed.
\end{abstract}

Keywords: correlation, nonlinear fringe-adjusted JTC, face recognition.

\section{INTRODUCTION}

In recent years, research on optical correlation techniques (OCT) for pattern recognition has gained significant momentum owing to their remarkable discrimination power and ability of detection and estimation of target objects [1]. Its generic principle consists to compare a target image with one or many reference images of a learning database. OCT have been investigated intensely for their scientific and technological potentials in the 1980s and 1990s. At the turn of the $20^{\text {th }}$ century the subject of OCT seemed to have reached a sort of maturity. All-optical implementations are rather complex to develop. These approaches, however, are not the universal panacea and have their drawbacks and stringent requirements, i.e. aberration effects, alignment of components, limitation of the overall speed by how fast the information can be updated on the input and output devices, and need of a costly optoelectronic interface. Furthermore, use of all-optics cannot be justified for many applications, especially when the target image size is small. We note also that while an all-optical approach can be well controlled, it has also severe limitations as was shown in a recent work dealing with a surveillance video system which detects various posture-based events [2], i.e. the target image size should be small and the video rate should not be excessive. Thus, alternate hybrid (numerical-optical using optoelectronic interface) methods have been proposed as substitutes for all-optical techniques. Within this context, hybrid techniques, 
i.e. numerical implementation of correlation, have been considered an alternative to all-optical methods because they show a good compromise between performance and simplicity. Although hybrid techniques have generated many impressive results for the problem of correlation, a numerical approach frequently requires some adaptation of the correlation method in order to optimize the resources of the numerical target. On the other hand, an all-numerical implementation leads to a larger applicability, reconfigurability, and adaptation of the correlation pre-processing to the considered application. We have recently proposed a correlation method for face recognition applications which can be implemented using an all-numerical and reprogrammable target such as the graphics processor unit (GPU) [3]. Other authors have considered field-programmable gate arrays (FPGA) [4].

Our goal is to increase correlation discrimination by considering a numerical implementation of correlation. In the present work, we examine an all-numerical image processing based on the far-field Fraunhofer diffraction [5]. We detail the theoretical methods to design a Vanderlugt correlator (VLC) using either the fast Fourier transform (FFT) algorithm, or a numerical implementation of the optical FT (NO_FT). Next, we present the results and related discussions demonstrating several advantages of the NO_FT compared to the FFT. We conclude with a brief summary and a discussion of some of the wider implications of these results.

\section{NUMERICAL IMPLEMENATION OF THE FOURIER TRANSFORM BASED ON THE FRAUNHOFER DIFFRACTION}

Before delving into the details of the VLC, sometimes referred to as the $4 \mathrm{f}$ correlator, we must first briefly review the algorithmic principle of OCT via the VLC as illustrated in Fig. (1).

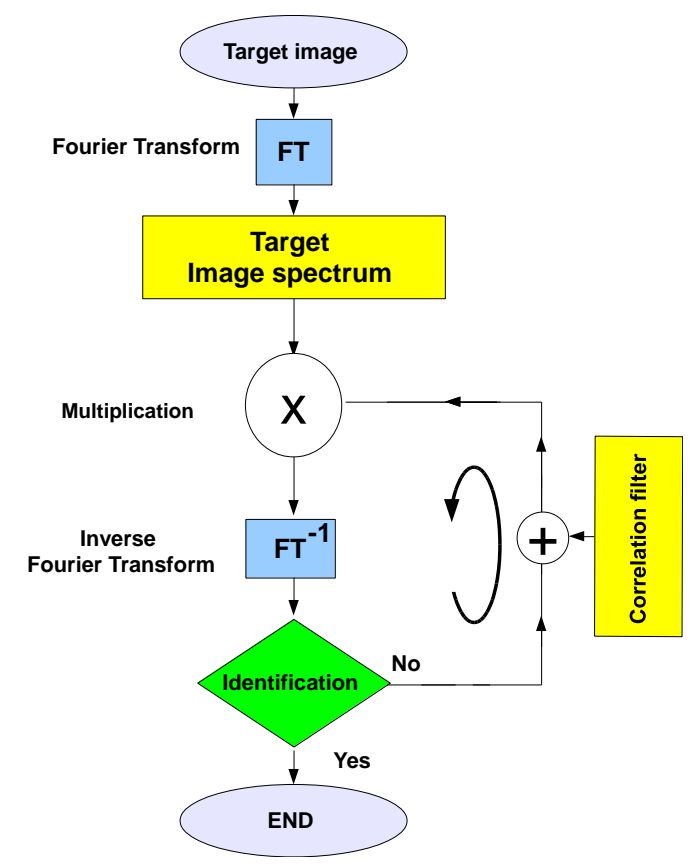

Fig. 1 : Illustrating the algorithm principle of OCT.

It is based on the multiplication between the spectrum, obtained by Fourier transforming the target image, with a correlation filter made from the FT of a reference image and placed in the Fourier plane. The correlation plane is obtained from $\mathrm{FT}^{-1}$ of the product of both spectra. This results in a more or less intense central correlation peak depending on the degree of similarity between the target object and the image reference. The correlation filter was fabricated from a reference image according a specific scheme [1]. In what follows, we used a phase only filter (POF). 
To determine the degree of similarity between the object function and the filter, we used the peak-to-correlation energy (PCE) efficiency criterion [1].

Notably, all correlations of objects with a filter examined in this work rely upon the computation of one FT and one $\mathrm{FT}^{-1}$. This is accomplished numerically. FT computation algorithms have been studied extensively in recent years, e.g. FFT. FFT is an efficient algorithm to compute the discrete Fourier transform (DFT) and its inverse, but cannot compute an optical FT. Here, we will focus on the Fraunhofer diffraction to implement numerically the FT, i.e. (NO_FT).

The next avenue of pursuit employs the Fourier optical analysis developed in [5]. The paraxial diffraction integral for propagating a field through a given distance in free space is obtained by the Fraunhofer approximation. Suppose that a monochromatic wave is incident on a finite aperture in an infinite opaque screen. The expression for the field amplitude at point $(u, v)$ is

$$
U_{i}(u, v)=\frac{\exp (j k z)}{j \lambda z} \exp \left(j \frac{k}{2 z}\left(u^{2}+v^{2}\right)\right) \iint_{\square^{2}} U_{o}(x, y) P(x, y) \exp \left(-j \frac{2 \pi}{\lambda z}(u x+v y)\right) d x d y
$$

where $k=2 \pi / \lambda$ denotes the wave vector, $\lambda$ is the wavelength of the incident wave illuminating the transparency. If we suppose that $z$ is chosen to be so large that the phase factor $P(x, y)=1$ over the entire region of the $(x, y)$ plane in which $U_{\mathrm{o}}(x, y)$ is non-zero, $U_{\mathrm{i}}(u, v)$ is just the two-dimensional FT of the aperture $U_{\mathrm{o}}(x, y)$, except for a multiplicative phase factor preceding the integral and which does not affect the intensity of the diffracted light, at frequencies $\left(\frac{u}{\lambda z}, \frac{v}{\lambda z}\right)$. This is called the Fraunhofer approximation. Now, one of the most remarkable properties of a convergent lens is its ability to perform a two-dimensional FT. If we place the object to be transformed directly against a thin convex lens of focal length $f$, the distribution of field amplitude across the back focal plane of the lens, Eq. (1) can be applied by putting $z=f$. As an example, we consider the special case of a circular aperture with diameter $w_{l}$. Then, we have

$$
P(x, y)=\operatorname{circ}\left(\frac{\sqrt{x^{2}+y^{2}}}{w_{l}}\right)
$$

where the circle function $\operatorname{circ}(x)=1$ if $x<1,=1 / 2$ if $x=1$, and 0 if $x>1$. A MATLAB function can compute the Fraunhofer diffraction pattern using the algorithm discussed in [5]. Fig. 2 shows the result from this computation for a circular aperture of diameter $w_{l}=\ldots \mathrm{mm}$ illuminated with light of wavelength ... nm. The Fraunhofer diffraction pattern is calculated at distance of $z=0.25 \mathrm{~m}$.

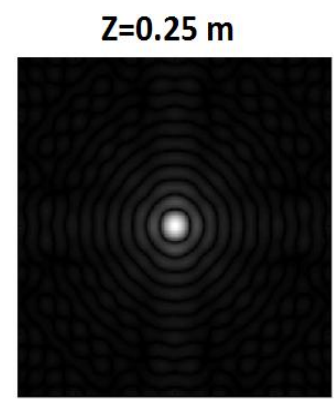

Fig. 2 : Fraunhofer diffraction pattern of a circular aperture.

\section{NUMERICAL VANDERLUGT CORRELATOR BASED ON FFT AND FRAUNHOFER DIFFRACTION}

We now consider a numerical implementation of a VLC. We test this scheme by considering the correlation of the image shown in Fig. 3 (a) with a POF fabricated with a reference image which is identical to the target image. A 
representation of this filter is displayed in Fig. 3 (b). Fig. 3(c) shows the correlation plane obtained by following the algorithm described in section 2. The corresponding 3D correlation plane is shown in Fig. 3 (d). It can be seen that the correlation peak is centred in the output Fourier plane. Note, however, that this peak vanishes if the reference image is different from the target image (not shown). In addition, we duplicated this calculation by using the FFT instead of the NO_FT. Significant differences were observed which are next discussed in depth.

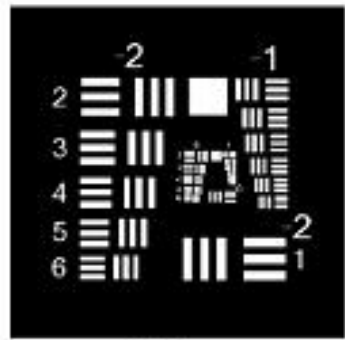

(a)

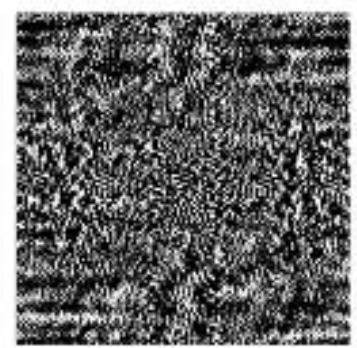

(b)

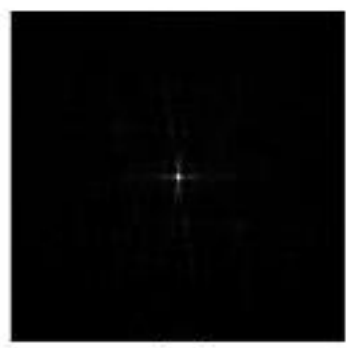

(c)

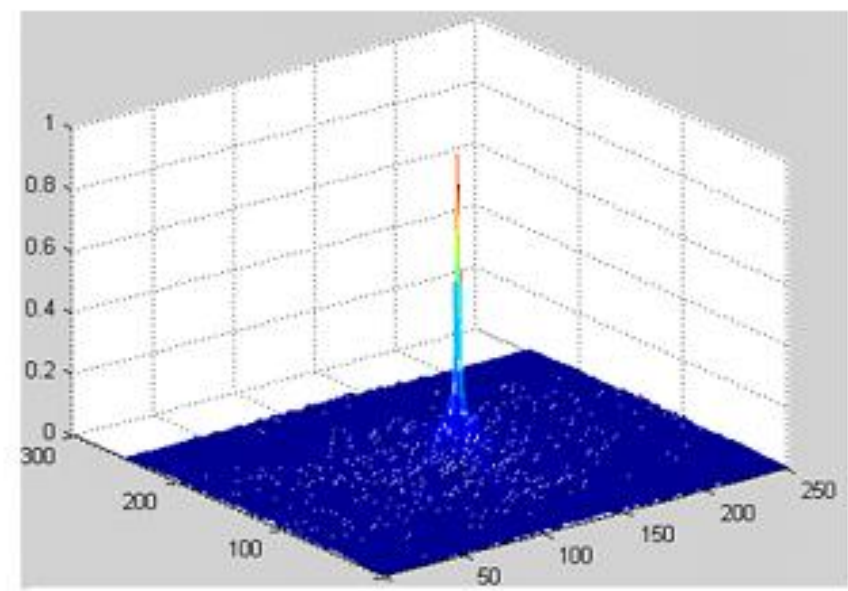

(d)

Fig. 3 : 3D correlation plane obtained by correlating the image in (a) with the POF shown in (b).

\section{COMPARISON OF PERFORMANCES}

As previously mentioned we compare the VLC results using a five-reference composite POF [1] using either FFT or NO_FT algorithms. Robustness and discrimination capability against horizontal and vertical rotations of the target image with respect to the reference images are systematically tested. For that purpose, we use different faces with several viewpoints from the Pointing Head Pose Image Database (PHPID) [6]. Fig. (4) shows an example of this base for subject 4 (hereafter, noted P4) where horizontal rotations within the range $\left[-90^{\circ}, 90^{\circ}\right]$ and vertical rotations within the range $[-$ $\left.15^{\circ},+15^{\circ}\right]$ are considered. The reference images used for fabricating the composite filter, i.e. POF 5_Refs, are 5 images of $\mathrm{P} 4$ with angle of vertical rotation set to $0^{\circ}$ and five horizontal rotation angles $\left(-45^{\circ},-15^{\circ},+15^{\circ},+30^{\circ}\right.$, and $\left.+45^{\circ}\right)$, i.e. P4_0_(-45) ; P4_0_(-15) ; P4_0_(+15) ; P4_0_(+30) ; P4_0_(+45). 


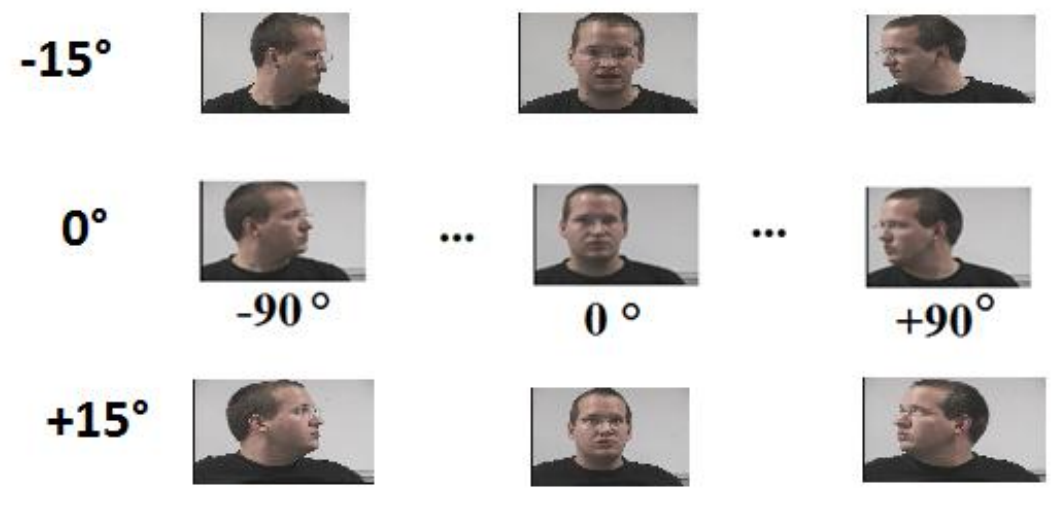

Fig. 4: Examples of horizontal and vertical facial rotations for subject 4 (P4) from the PHPID [6].

Fig. 5 shows the correlation planes obtained from the two methods. Three examples of correlation planes are displayed in $\mathrm{a}, \mathrm{b}$, and $\mathrm{c}$ by correlating images of subject $\mathrm{P} 4$ (rotation angles $\left(-15^{\circ},+30^{\circ}\right),\left(0^{\circ},-30^{\circ}\right)$, and $\left(+15^{\circ}, 0^{\circ}\right)$ ) with the 5-reference composite filter POF 5_Refs using the FFT algorithm. Fig. 5 (d, e, and f) show the corresponding correlation planes using the NO_FT algorithm.

Now we make a few observations on what we have learned. Firstly, it can be seen that the correlation peaks are higher and narrower when the NO_FT algorithm is used. Remarkably, it should be noted that no correlation peak can be found when the FFT algorithm is used for the case with rotation angles $(+15,0)$, while the peak is clearly visible for NO_FT. This numerical study demonstrates the robustness of the NO_FT method. Secondly, discrimination tests were also performed with three other subjects. Importantly, our conclusions are essentially unchanged. Thirdly, The ROC curves in Fig. 6 demonstrate also the effectiveness of the NO_FT for image recognition. The ROC curve can be represented by plotting the fraction of true positives out of the positives TPR (sensitivity, or true positive rate) versus the fraction of false positives out of the negatives FPR (1- specificity, or false positive rate) [7]. Fig. 6 (a) shows the ROC curve obtained using the MATLAB function of FFT. The corresponding ROC curve obtained with the NO_FT algorithm is shown in Fig. 6(b). The results of our tests clearly show the good recognition performance of the VLC using NO_FT, i.e. $\mathrm{TPR}=0.71$ corresponding to $\mathrm{FPR}=0$. 


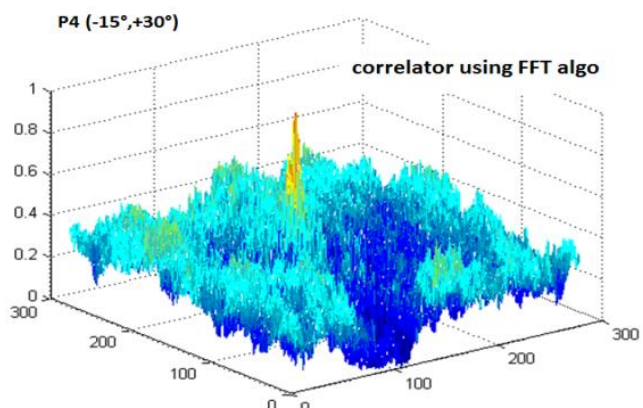

(a)

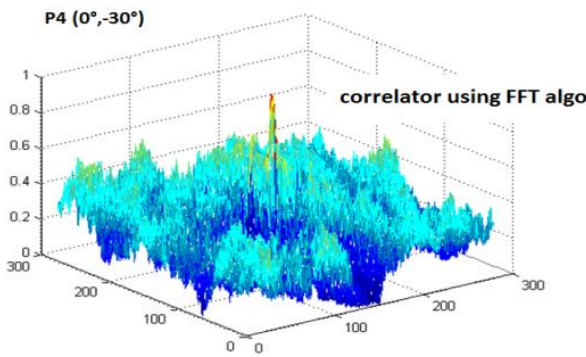

(b)

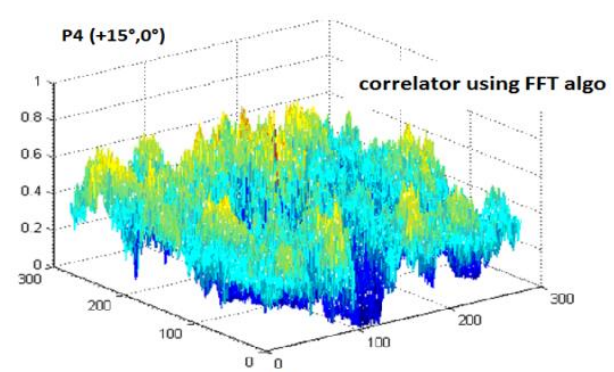

(c)

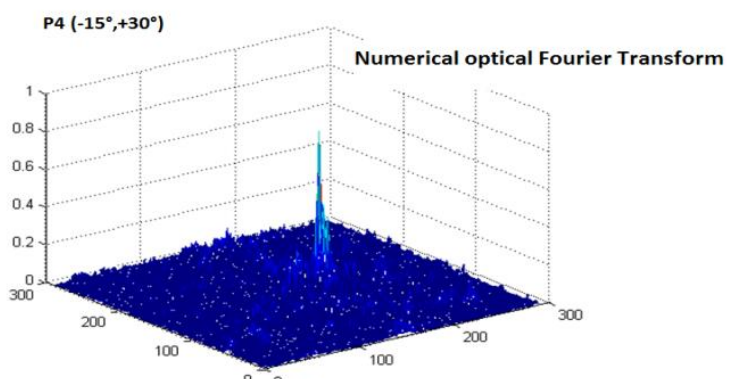

(d)

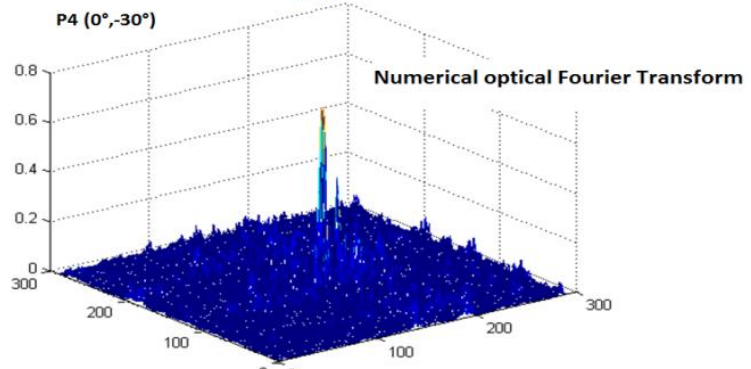

(e)

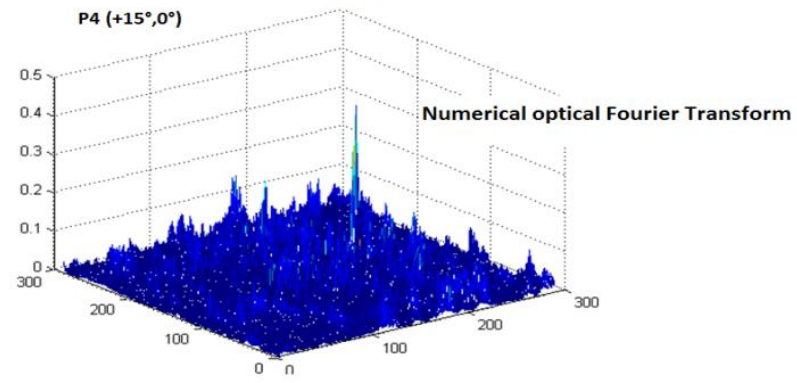

(f)

Fig. 5: 3D correlation planes obtained by using: (a, b, and c) the FFT algorithm; (d, e, and f) the NO_FT.

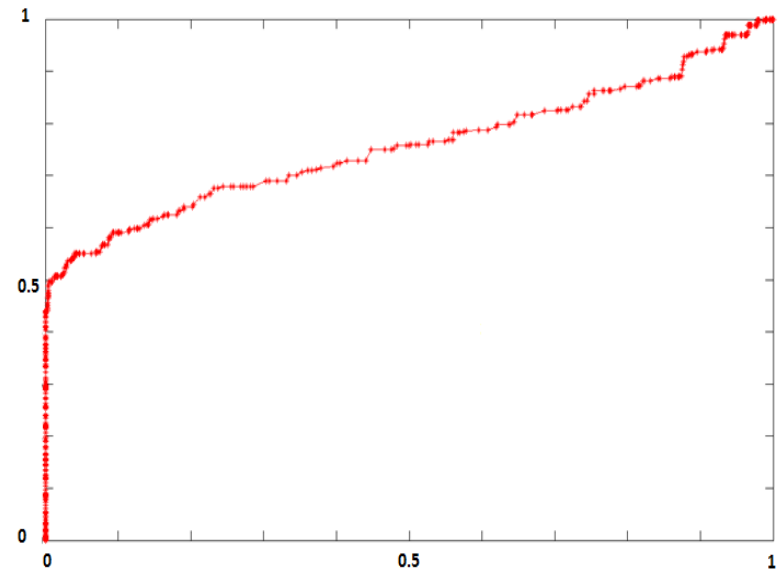

(a)

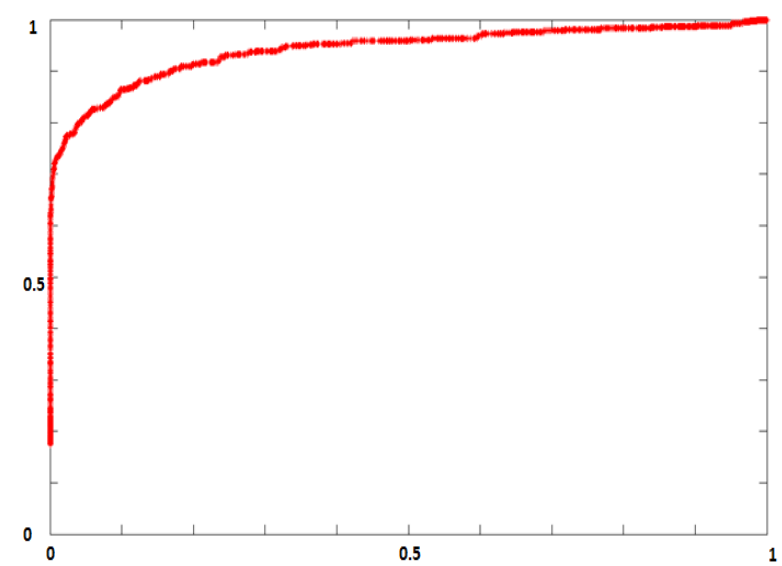

(b)

Fig. 6: ROC curves obtained by using: (a) the FFT algorithm; (b) the NO_FT. 


\section{CONCLUSION}

In this work we suggest a method to compute the NO_FT which is used for implementing numerically a VLC. Based on these numerical calculations, we demonstrate that NO_FT is robust and highly discriminating. In comparison with the results using FFT correlation discrimination is significantly improved. The next step is clearly to perform optically the analysis suggested here, and we have begun this task using spatial light modulators.

\section{REFERENCES}

[1] A. Alfalou and C. Brosseau, "Understanding Correlation Techniques for Face Recognition: From Basics to Applications," Face Recognition, Milos Oravec (Ed.), ISBN: 978-953-307-060-5, INTECH. Available online from: http://sciyo.com/articles/show/title/understanding-correlation-techniques-for-face-recognition-from-basics-to applications.

[2] M. Elbouz, A. Alfalou, and C. Brosseau, "Fuzzy logic and optical correlation-based face recognition method for patient monitoring application in home video surveillance", Opt. Eng. 50, 067003 (2011).

[3] Y. Ouerhani, M. Jridi, and A. Alfalou "Fast Face Recognition Approach Using a Graphical Processing Unit "GPU". Porc. IEEE IST: International Conference on Imaging Systems and Techniques, 80-84 (2010).

[4] Special Issue on: "Field-Programmable Technology", J. Real-Time Image Proc. 2, (2011).

[5] D. G. Voelz, Computational Fourier Optics: A MATLAB Tutorial, SPIE Tutorial Texts TT89 (SPIE Press, Bellingham, WA, 2011). See also J. Goodman, Introduction to Fourier Optics (McGraw-Hill, San Francisco, 1968).

[6] N. Gourier, D. Hall, and J. L. Crowley, "Estimating Face Orientation from Robust Detection of Salient Facial Features," Proc. of Pointing 2004, ICPR, International Workshop on Visual Observation of Deictic Gestures (2004).

[7] J. P. Egan, Signal Detection Theory and ROC Analysis (Academic Press Series in Cognition and Perception, New York, 1975). 